\title{
CORRESPONDENCE
}

\section{Clinical experience of neuroanaesthesia trainees: A survey}

\section{Mark Anders, Joe Sebastian}

\section{INTRODUCTION}

The neuroanaesthesia syllabus for intermediate and higher modules involves exposure to a wide variety of clinical experiences from elective and emergency intracranial procedures, spinal surgery, interventional radiology and anaesthesia for imaging such as magnetic resonance imaging (MRI).

Some centres have designed a minimum clinical caseload for trainees (such as a minimum number of craniotomies), whereas others have no such targets.

Monitoring this experience is important to assess all neurosurgical sub-specialties that are adequately covered.

\section{Aims}

The aims of this audit were to assess adequacy of clinical exposure of neuroanaesthesia trainees in the North West deanery who were undergoing their intermediate and higher modules over a period of 1 year. These were compared to the syllabus for the relevant module to ensure that these areas were adequately being covered.

\section{Syllabus criteria}

Trainees undertake their first neuroanaesthesia module during their intermediate stage of training: A 2-year period of experience which follows basic training and passing the Primary Fellowship of the Royal College of Anaesthetists (FRCA). Neuroanaesthesia is then revisited for a higher module, following passing the final FRCA, during the next level of training.

The Royal College of Anaesthetists (RCoA) 2010 syllabus for neurosurgery, neuroradiology and critical care includes knowledge areas and skill areas for attainment. ${ }^{[1]}$

\section{Salford Royal Foundation Trust, United Kingdom}

Address for correspondence:

Dr. Mark Anders, Department of Anaesthesia,

Salford Royal Hospital Trust, Salford, Stott Ln,

Salford M6 8HD, United Kingdom.

E-mail: markanders@doctors.org.uk
The areas of learning that should be assessable are as follows.

Intermediate experience should include:

- Elective and emergency intracranial surgery

- Shunt surgery

- Cervical spinal surgery

- Lumbar spinal surgery

- Prone and lateral positioning

- Neuroradiology

- Simple emergencies (e.g., external ventricular drain) with distant supervision.

Higher module trainees should take part in:

- Routine and emergency surgery

- Perioperative care for complex spinal surgery

- Leadership of neuroanaesthesia emergencies

- Complex intracranial and spinal surgery

- Neuroradiological investigations

- Indirect supervision of emergency non-complex intracranial/spinal surgery.

\section{Standards}

Trainees should meet the requirements of the RCoA syllabus for their modules.

Therefore,

- $100 \%$ of intermediate trainees should have experience in the above areas and have performed simple emergency cases with distant supervision

- $100 \%$ of higher trainees should have experience in the above areas and have experience with distant supervision in non-complex intracranial and spinal surgery.

\section{SUBJECTS AND METHODS}

All trainees who had completed a module in the last year were contacted. They completed an online questionnaire or submitted a case list from their module. The case lists were then compared to the relevant curriculum.

\section{RESULTS}

A total of 24 complete case lists were gained with a further 9 partial lists [Table 1].

The mean number of intermediate module cases was 51 , with a range of 36-84 cases. The mean number of higher cases was 54 with a range of 36-93.

The most represented areas were 'simple' craniotomies with a mean number of 10 (intermediate) and 8 (higher) cases together with non-instrumented spinal surgery (13 and 11). 
Table 1: Number of cases in each area of neuroanaesthesia during the module

\begin{tabular}{lcc}
\hline & $\begin{array}{c}\text { Intermediate } \\
\text { module } \\
\text { mean (range) }\end{array}$ & $\begin{array}{c}\text { Higher } \\
\text { module } \\
\text { mean } \\
\text { (range) }\end{array}$ \\
\hline Elective craniotomy & $14(7-21)$ & $10(4-20)$ \\
Endoscopic & $2(0-9)$ & $2(0-3)$ \\
Other elective & $3(0-9)$ & $4(0-10)$ \\
Simple emergency & $2(0-6)$ & $6(1-22)$ \\
Burr-hole & $1(0-4)$ & $5(2-15)$ \\
Emergency craniotomy & $1(0-6)$ & $3(1-35)$ \\
Other emergency & $1(0-2)$ & $3(0-3)$ \\
C spine & $6(2-14)$ & $7(3-13)$ \\
Thoracolumbar & $15(7-41)$ & $14(5-30)$ \\
NXR & $5(2-12)$ & $2(0-5)$ \\
Magnetic resonance imaging & $2(0-8)$ & $1(0-5)$ \\
Total & $51(36-84)$ & 54 \\
& & $(36-93)$ \\
\hline
\end{tabular}

Inclusive of all craniotomies, intermediate trainees experienced a mean of 14.8 craniotomy cases and higher trainees 13.7 cases. However, one of the higher trainees only saw five craniotomies in total.

The least-represented areas were awake craniotomies (11/24 trainees had experience), clippings (15/24) and anaesthesia for MRI (15/23).

All trainees were involved in anaesthetising for cervical spine surgery, and all but one for instrumented thoracolumbar surgery.

All of the trainees on their intermediate module spent time in neuroradiology and experienced at least one coiling. Two of the nine higher trainees did not have neuroradiology experience during their module.

8/12 intermediate trainees directly experienced emergency shunt surgery. 9/12 intermediate trainees experienced emergency intracranial operations.

In terms of case supervision, $90 \%$ of neuroanaesthesia cases were directly supervised by a senior anaesthetist for the intermediate trainees and $66 \%$ for the higher trainees. All but one of the higher trainees gained experience performing multiple anaesthetics (range 7-27 cases) with local or distant supervision. All but two of the intermediate trainees performed some cases (1-13 cases) with local or distant supervision.
Positioning of the patient was not recorded specifically in most case lists, so assessing the experience in this area was not possible.

Overall case mix was as follows for the two modules [Figures 1 and 2].

A table showing the percentage of trainees achieving the set standards.

Intermediate
$100 \%$ of trainees gained cervical and thoracolumbar
experience
$100 \%$ of trainees gained neuroradiology experience
$100 \%$ of trainees gained elective craniotomy experience
$100 \%$ of trainees anaesthetised prone-positioned patients
$82 \%$ anaesthetised for simple cases without immediate
supervision
$75 \%$ of trainees gained emergency craniotomy experience
Higher
$100 \%$ of trainees took part in elective and emergency
surgery
$100 \%$ of trainees anaesthetised for complex spinal
surgery
$90 \%$ anaesthetised cases without immediate or local
supervision
$78 \%$ anaesthetised for neuroradiology procedures

A table showing specific comments from responders

'Difficult areas to gain experience in'

Awake craniotomy, most brain surgery

NXR, neurovascular

Element of luck as to how many craniotomies you see. Not able to see any awake craniotomies due to logistics of when they were happening/on-call commitments/leave, etc.

$\mathrm{MR} /$ radiology

I got a fair mix of cases and did not really have to request anything in particular

Awake craniotomy happens infrequently

'Possible improvements to delivery of module'

I asked specifically to do some spinal lists to gain

knowledge on positioning and cell salvage

Difficult to accommodate everyone when so many trainees are doing neuromodules at the same time

More lists?

A teaching session in theatre which instructs us in proper positioning for the spinal cases would be useful pre-module

No - I felt that my list allocation took into account my requests 


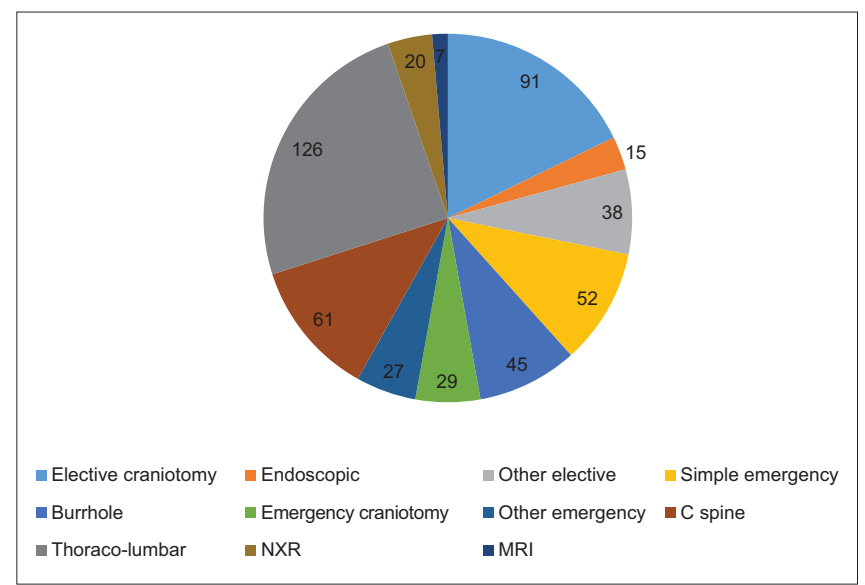

Figure 1: Proportional case mix for higher module with totals including all trainees

\section{DISCUSSION}

This audit has shown that the majority of trainees have no difficulty in attaining sufficient numbers for their module.

All trainees experienced some spinal surgery. For the survey, this was divided into cervical, instrumented thoracolumbar and non-instrumented thoracolumbar. The most difficult area of these three to gain experience in was instrumented spinal surgery. However, all higher trainees and all but one of the intermediate trainees had experience in all three areas.

Those trainees doing their higher module only had on average three more cases per attachment, but the cases mix tended to include much more emergency cases such as burr-hole evacuation of subdural haematomas.

The logbook areas where trainees experienced greater difficulties in gaining experience (awake craniotomies, clippings and anaesthesia for MRI) are areas where direct experience is not essential for either module, but knowledge and experience gained will still apply to other areas of the syllabus.

Responders to the survey felt that the most difficult areas to gain experience in were craniotomies, especially awake craniotomies, and neuroradiology, which is reinforced by the results above. MRI was also reported as one of the most difficult areas to gain experience in. Of these areas, neuroradiology is specifically present in the syllabus and this could be reinforced during future pre-module meetings.

The main improvement listed by responders actually involves spinal lists, with two people requesting teaching in proper positioning prior to the module starting. This could be included in future inductions.

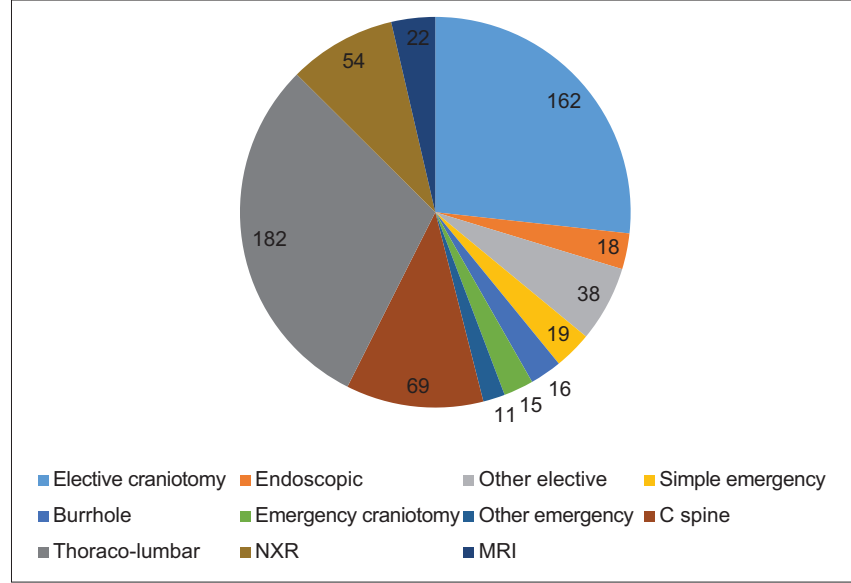

Figure 2: Proportional case mix for intermediate module with totals including all trainees

Using a log-book to monitor trainee progress has been previously carried out in surgery, ${ }^{[2,3]}$ but little has been published as to how they can be used in anaesthesia. On-going monitoring will be useful in maintaining delivery of the neuroanaesthesia syllabus and preventing any gap in experience.

\section{Future improvements}

- Reinforce the importance of neuroradiology at start of modules

- Include teaching of prone positioning.

\section{Limitations}

This was a survey-based audit so is limited by response rates from past trainees. It may have been that those who participated have logbooks that are not representative of the entire group of trainees.

It also relies on accurate completion of the logbook with cases needing to be correctly entered.

Some of the information required by the syllabus cannot be found from logbooks directly, e.g., patient positioning.

\section{Financial support and sponsorship} Nil.

\section{Conflicts of interest}

There are no conflicts of interest.

\section{REFERENCES}

1. Royal College of Anaesthetists 2010. Curriculum for a CCT in Anaesthetics; 2010. Available from: http://www.rcoa.ac.uk/ careers-training/training-anaesthesia/the-training-curriculum/ CCT2010. [Last accessed on 2015 Jan 15].

2. Lonergan PE, Mulsow J, Tanner WA, Traynor O, Tierney S. Analysing the operative experience of basic surgical trainees in Ireland using a web-based logbook. BMC Med Educ 2011;11:70. 
3. Beasley SW, McBride C, Pearson ML. Use of the operative logbook to monitor trainee progress, and evaluate operative supervision provided by accredited training posts. Surgeon 2011;9 Suppl 1:S14-5.
This is an open access article distributed under the terms of the Creative Commons Attribution-NonCommercial-ShareAlike 3.0 License, which allows others to remix, tweak, and build upon the work non-commercially, as long as the author is credited and the new creations are licensed under the identical terms.

\begin{tabular}{|l|l|}
\hline \multicolumn{2}{|c|}{ Access this article online } \\
\hline Quick Response Code: & Website: \\
\hline & www.jnaccjournal.org \\
\cline { 2 - 2 } & \\
\hline
\end{tabular}

